EDITORIAL

\title{
Quality Assurance (QA) in Laboratory Testing
}

\author{
Md. Tahminur Rahman
}

\author{
Prof. Dr. Md. Tahminur Rahman, Professor \& Head, Pathology \\ Anwer Khan Modern Medical College, Dhanmondi, Dhaka \\ email: tahminurrahman@yahoo.com
}

Government of Bangladesh has recently approved a draft on National Health Policy with an aim to reach the minimum Health care facility (MDG) by $2015^{1}$.

The purpose of the health care system in a country is to correctly diagnose the disease, identify the factors responsible for the disease and take appropriate preventive and curative measures to control the disease. The pathologists and laboratory people are very much involved in the correct diagnosis, effective treatment and follow up of the patients. For correct diagnosis quality assurance and quality control are very important. A test wrongly done is worst than a test not done. Quality control is the process to ensure prevent a test from being done wrongly. Laboratory quality control is designed to detect, reduce and correct deficiencies in laboratories analytical process to release patient results and improve the quality of test result ${ }^{2}$.

Quality assurance (QA) is aimed at ensuring quality test results. The purpose of quality assurance is to give relevant, reliable, timely test results which is interpreted correctly. Quality assurance involves activities both inside and outside laboratory, good laboratory practice and proper management skilll. The WHO definition of quality assurance is a total process whereby the quality of lab reports can be quaranteed ${ }^{3}$.

It has been summarised as the

- right result, at the

- right time, on the

- right specimen, from the

- right patient, with result/interpreatation based on

- correct reference date, and at the

- right place
Quality control (QC) or the other hand covers the part of quality assurance which primarily concerns the control of errors in the performance of tests and verification of test results. Quality control must be practical, achievable and affordable. The primary aim of quality control is to do the test reliably. The broad aim of quality control is that results from one lab should be comparable with that from any lab in the world provided the same method is followed ${ }^{2}$.

Quality control can be implemented in two ways:

a. Internal or intra laboratory QC-performed by individual labs at their own levels. It forms the day to day basis working quality assurance.

b. External or inter laboratory QC-performed by many labs at the same time, monitored by one. It is costly.

Many international agencies supervise quality assurance namely ISO, ioQA, intertek and Quality assurance program run by different reputed laborites aboard. Proper QA dramatically improve the effectiveness of QC program of a laboratory ${ }^{4,5,6}$.

For histo and cytopathology quality control involves intra departmental consultation, random case review, intra departmental and inter departmental conference, inter institutional review, specimen adequacy record, lost specimen record, turn about times, techniques i.e staining, proper sectioning, embedding, fixation, re and de hydration of samples, clearing, mounting, labelling etc ${ }^{7,8,9}$.

For biochemistry quality control involves optimum condition variance (OCV), routine condition variance known value (RCVK), composite and logn term assay, computer aided check, accuracy and precision tests. Accuracy is defined as 
closeness of the test result and accepted true value, whereas precision is a measure of reproducibility. Daily QC chart preparation is mandatory. Control value within $\pm 2 \mathrm{SD}$ is good sign and patient's results obtained are reliable and can be reported. If control value is beyond \pm 2 SD the result must not be reported and fresh control serum should be measured together with few patients sample. If the result is now within $\pm 2 \mathrm{SD}$, the result can be reported. The type of material used for QC are frozen, pooled serum; commercially available lyophilized freeze dried pool serum; commercially available low temperature liquid serum pools ${ }^{7}$.

For microbiology quality control involves use of ATTCC strains of organisms for standardization, measuring the disc potency, MIC, media contamination, standardization of staining procedures, autoclaving and sterilizaiton of media, Safe disposal of infected materials is also an important procedure to maintain standard lab proactice.

For hematology standardization of staining procedures, preparation of proper blood film, bone marrow smear, special staining techniques flow cytometry, chromosome analysis are the mainstay of quality conrol.

Wrong test results are caused by faults on the part of the clinicians like sending samle without identificaiton number and name of the test, collection date, appropriate container, sending specimens divided in several parts to different labs; without clinical history, provisional diagnosis, other relevant test results, radiology/imaginf reports/etec.

Wrong results are also caused by the faults of laboratory and the pathologists which can be divided into preanalytical stage faults, analytical stage faults and post analytical stage faults. In the preanalytical stage the faults are misidentification of patients due to incomplete ID, incorrectly labelled specimen container, partly erased or illegible label and disparity between request form and specimen.
Faults may also be with the specimen. A faulty specimen is one which is inadequate, collected at incorrect time in dirty and contaminated container, in a container without proper anticoagulant and stored incorrectly or in hemolysed status. These are known as pre analytical faults.

In the analytical stage the faults resulting in wrong findings if the principle and procedure of the test is not strictly followed; reagents, standards, QC materials are not prepared, mixed, processed properly and performance standards are not followed strictly.

In the post analytical stage the faults may occur if reporting, checking and verifications are not done properly, interpretation of test results are not considered seriously, and abnormally/unexpected result is not taken seriously and not reviewed and or performed again.

It is very important to maintain QA and QC for reliable, quick and dependable results in shortest possible time. This will help the clinicians to come to a correct diagnosis and treat the patient early. This will lead to early recovery and save working time, money for the patient and the nation. But to achieve this all the relevant parties involved ie the clinicians, pathologists, patients all have equal responsibilities.

To monitor QA and QC, minimize errors establishment of a Central Reference Laboratory and Institute of Pathology is a must and need of the hour. The sooner the Government, peoples representatives, Medical professionals, Social workers, Journalists, Patients and their relative understand this, the better is the out come. Bangladesh Society of Pathologists should come up with concrete proposals for this and talk to the Government and other appropriate agencies for its early implementation. The good news is that Government has accepted in principle the concept of a Central reference laboratory, allocated budget for this project and preliminary baseline work has been done. Now the society of Pathologists should work shoulder to shoulder with Ministry of Health for quick implementation of the project. 


\section{Reference}

1. National Health Policy January 2010, Final Draft Prepared by Ministry \& Health \& Family Welfare Govt. of Bangladesh.

2. Kumar V, Abbas AK, Fausto N Robbins \& Cotran pathologic Basis of Disease.ed. $7^{\text {th }} 2004$ Saunders USA.

3. Rosai. J. Rosai and Ackerman's Surgical Pathology ed. $9^{\text {th }}$ ed. Mosby USA; 2004: 2790-2799.

4. Laboratory Quality Control wikipedia, www eu. wikipedia. org/wiki/Lab. quality control.
5. Quality assurance ioglobal. net www. ioglabal.net.

6. Quality Control Testing labortory www. interetckcb.com/analytical/gelab. shtml.

7. Talib VH, A hand book of medical laboratory technology. $2^{\text {nd }}$ ed2002, CBS publishers and distributors, India: 227-32.

8. Internet search on quality control and quality assurance.

9. Gowenlock AH, McMurry JR and McLauchian DM. Varley's clinical biochemistry; $1^{\text {st }}$ Indian edition, CBS publishers and distributors India; reprint 2002: 285-300. 